\title{
Diversity of New Three-Wave Solutions and New Periodic Waves for the $(3+1)$-Dimensional Kadomtsev-Petviashvili-Boussinesq-Like Equation
}

\author{
Meiyu Li', Sudao Bilige ${ }^{1 *}$, Runfa Zhang², Lihui Han' \\ ${ }^{1}$ Department of Mathematics, Inner Mongolia University of Technology, Hohhot, China \\ ${ }^{2}$ School of Software Technology, Dalian University of Technology, Dalian, China \\ Email: *inmathematica@126.com
}

How to cite this paper: Li, M.Y., Bilige, S., Zhang, R.F. and Han, L.H. (2020) Diversity of New Three-Wave Solutions and New Periodic Waves for the $(3+1)$-Dimensional Kadomtsev-Petviashvili-Boussinesq-Like Equation. Journal of Applied Mathematics and Physics, 8, 2142-2156. https://doi.org/10.4236/jamp.2020.810160

Received: September 7, 2020

Accepted: October 17, 2020

Published: October 20, 2020

Copyright $\odot 2020$ by author(s) and Scientific Research Publishing Inc. This work is licensed under the Creative Commons Attribution International License (CC BY 4.0).

http://creativecommons.org/licenses/by/4.0/

\begin{abstract}
Based on the generalized bilinear method, diversity of exact solutions of the $(3+1)$-dimensional Kadomtsev-Petviashvili-Boussinesq-like equation is successfully derived by using symbolic computation with Maple. These new solutions, named three-wave solutions and periodic wave have greatly enriched the existing literature. Via the three-dimensional images, density images and contour plots, the physical characteristics of these waves are well described. The new three-wave solutions and periodic solitary wave solutions obtained in this paper, will have a wide range of applications in the fields of physics and mechanics.
\end{abstract}

\section{Keywords}

KPB-Like Equation, Generalized Bilinear Form, New Three-Wave Solutions, New Periodic Wave

\section{Introduction}

The nonlinear integrable systems, which are of great importance in the field of physics and mathematics [1], have been paid more and more attention and many exact solutions of the nonlinear integrable systems are obtained by using different effective methods. In recent, experts and scholars have become more interested in the research of solutions to nonlinear partial differential equations (NPDEs). They have extensively expanded the field of NPDEs in many aspects. The Hirota bilinear form plays an important role in presenting soliton solutions, although some intelligent guessing is usually required. In recent years, more ex- 
act solutions have been found for solving integrable equations, such as solitary wave [2] [3] [4] [5] [6], rogue waves [7] [8] [9], periodic solitary wave [10] [11] [12], optical soliton [13], lump solution and interaction solution [14]-[34], and cross-kink wave solution [35]. It is natural and interesting to search for new three-wave solutions and new periodic wave to NPDEs taking advantage of hirota bilinear forms.

The $(3+1)$-dimensional Kadomtsev-Petviashvili-Boussinesq-like (KPB-like) equation is usually written as [36]:

$$
\mathrm{P}_{\mathrm{KPB}-\text { like }}(u): u_{x x x y}+3\left(u_{x} u_{y}\right)_{x}+u_{t y}+u_{x t}+u_{t t}-u_{z z}=0 .
$$

The generalized Kadomtsev-Petviashvili-Boussinesq equation is first proposed in [37]. Recently, based on bilinear Bäcklund transformation, some classes of exponential and rational traveling wave solutions of Equation (1) are presented [36]. And by using the Hirota bilinear method, the lump solutions of Equation (1) are obtained [38].

In the present paper, we will give Hirota bilinear from and generalized lilinear from of the KPB-like equation in Section 2. In Section 3, new three-wave solutions of the KPB-like equation will be obtained based on generalized bilinear from. In Section 4, new periodic wave solutions of KPB-like equation will be given, that supplement the existing literature on KPB-like equation. A few conclusion and outlook will be given in Section 5 .

\section{Bilinear Form}

Under dependent variable transformation:

$$
u(x, y, z, t)=2[\ln f(x, y, z, t)]_{x} .
$$

Equation (1) is transformed into the following generalized bilinear form with $p=3$,

$$
\begin{aligned}
& \mathrm{GB}_{\mathrm{KPB}-\mathrm{like}}(f) \\
& =\left(D_{p, x}^{3} D_{p, y}+D_{p, y} D_{p, t}+D_{p, t} D_{p, x}-D_{p, z}^{2}+D_{p, t}^{2}\right) f \cdot f \\
& =6 f_{x x} f_{x y}+2\left(f_{t y} f-f_{y} f_{t}\right)+2\left(f_{x t} f-f_{x} f_{t}\right)-2\left(f_{z z} f-f_{z}^{2}\right)+2\left(f_{t t} f-f_{t}^{2}\right) \\
& =0,
\end{aligned}
$$

where $p$ is an arbitrarily given natural number, often a prime number [39],

$$
\begin{aligned}
& D_{p, x_{1}}^{n_{1}} \cdots D_{p, x_{M}}^{n_{M}} a \cdot b\left(x_{1}, \cdots, x_{M}\right) \\
& =\prod_{i=1}^{M}\left(\frac{\partial}{\partial x_{i}}+\alpha \frac{\partial}{\partial x_{i}^{\prime}}\right)^{n_{i}} a\left(x_{1}, \cdots, x_{M}\right) b\left(x_{1}^{\prime}, \cdots, x_{M}^{\prime}\right) \times\left.\right|_{x^{\prime}=x_{1}, \cdots, x^{\prime}=x_{M}},
\end{aligned}
$$

where $n_{1}, \cdots, n_{M}$ are arbitrary non-negative integers, and for an integer $m$, the $m$ th power of $\alpha$ is computed as follows,

$$
\alpha^{m}=(-1)^{r(m)}, m \equiv r(m) \bmod p, 0 \leq r(m)<p .
$$

We can know that the following symbols are required: +1 or -1 .

If we take $p=3$, then we have

$$
\alpha_{3}^{1}=-1, \alpha_{3}^{2}=1, \alpha_{3}^{3}=1, \alpha_{3}^{4}=-1, \alpha_{3}^{5}=1, \alpha_{3}^{6}=1, \alpha_{3}^{7}=-1, \alpha_{3}^{8}=1, \alpha_{3}^{9}=1,
$$


which leads to

$$
\begin{aligned}
& D_{3, x} D_{3, t} f \cdot f=2 f_{x, t} f-2 f_{x} f_{t}, \\
& D_{3, y}^{2} f \cdot f=2 f_{y, y} f-2 f_{y}^{2}, \\
& D_{3, x}^{4} f \cdot f=6 f_{x, x}^{2}, \\
& D_{3, x}^{3} D_{3, y} f \cdot f=6 f_{x, x} f_{x, y},
\end{aligned}
$$

But if $p=2$, then we can get

$$
\begin{aligned}
& D_{2, t} D_{2, x} f \cdot f=2 f_{x t} f-2 f_{x} f_{t}, \\
& D_{2, y}^{2} f \cdot f=2 f_{y y} f-2 f_{y}^{2}, \\
& D_{2, x}^{4} f \cdot f=2 f_{x x x x} \cdot f-8 f_{x x x} f_{x}+6 f_{x x}^{2}, \\
& D_{2, x}^{3} D_{2, y} f \cdot f=2 f_{x x x y} f-6 f_{x x y} f_{x}+6 f_{x y} f_{x x}-2 f_{y} f_{x x x} .
\end{aligned}
$$

We have noticed that when $p=2$, the generalized bilinear form is transformed into Hirota bilinear form. Transform (2) is also a characteristic transformation for establishing Bell polynomial theory of soliton equation [40]. Its exact relation is as follows:

$$
\operatorname{GP}_{\text {KPB-like }}(u)=\left[\frac{\operatorname{GB}_{\text {KPB-like }}(\mathrm{u})}{f^{2}}\right] \text {. }
$$

Via the bilinear transformation (2), Equation (3) is transformed into

$$
\begin{aligned}
\mathrm{B}_{\mathrm{KPB}-\mathrm{like}}(u)= & \frac{3}{4} u_{x x} u v+\frac{9}{4} u_{x} u u_{y}+\frac{9}{8} u_{x} u^{2} v+u_{y t}+u_{t t}+u_{x t}+\frac{3}{8} u^{3} u_{y} \\
& +\frac{3}{8} u_{x} u_{y x}+\frac{3}{2} u_{x}^{2} v+\frac{3}{4} u^{2} u_{y x}+\frac{3}{2} u_{x x} u_{y}-u_{z z} \\
= & 0,
\end{aligned}
$$

where $u_{y}=v_{x}$. Hence, if $f$ solves the generalized bilinear the KPB-like Equation (3), the dimensionally reduced the KPB-like Equation (10) will be solved.

\section{New Three-Wave Solutions of the KPB-Like Equation}

To search for the new three-wave solution of the KPB-like Equation (1), we would like to start from an ansatz [41]

$$
f=\mathrm{e}^{-g}+k_{1} \mathrm{e}^{g}+k_{2} \cos (h)+k_{3} \sin (n)+a_{16},
$$

where $g=a_{1} x+a_{2} y+a_{3} z+a_{4} t+a_{5}, \quad h=a_{6} x+a_{7} y+a_{8} z+a_{9} t+a_{10}$, $n=a_{11} x+a_{12} y+a_{13} z+a_{14} t+a_{15}, \quad a_{i}(i=1,2, \cdots, 16)$ and $k_{i}(i=1,2,3)$ are real parameters to be determined later. Substituting (11) into Equation (3), we obtained a series of periodic wave solutions to the KPB-like equation with the help of Maple.

Case 1:

$$
\begin{aligned}
& a_{1}=\frac{a_{3}^{2}-a_{4}^{2}}{a_{4}}, a_{2}=0, a_{3}=a_{3}, a_{4}=a_{4}, a_{5}=a_{5}, a_{6}=0, a_{7}=\frac{\left(a_{3}^{2}-a_{4}^{2}\right) a_{9}}{a_{4}^{2}}, \\
& a_{8}=\frac{a_{3} a_{9}}{a_{4}}, a_{9}=a_{9}, a_{10}=a_{10}, a_{11}=\frac{\left(a_{3}^{2}-a_{4}^{2}\right) a_{14}}{a_{4}^{2}}, a_{12}=0, a_{13}=\frac{a_{3} a_{14}}{a_{4}}, \\
& a_{14}=a_{14}, a_{15}=a_{15}, a_{16}=a_{16}, k_{1}=k_{1}, k_{2}=k_{2}, k_{3}=k_{3},
\end{aligned}
$$


with the condition $a_{4} \neq 0$. Through the expression (11) and the transformation (2), we get the three-wave solution of the KPB-like equation

$u=\frac{2}{F}\left[-\frac{a_{3}^{2}-a_{4}^{2}}{a_{4}} \mathrm{e}^{-a_{4} t-\frac{x\left(a_{3}^{2}-a_{4}^{2}\right)}{a_{4}}-a_{3} z-a_{5}}+\frac{k_{1}\left(a_{3}^{2}-a_{4}^{2}\right) \mathrm{e}^{\xi_{1}}}{a_{4}}+\frac{k_{3}\left(a_{3}^{2}-a_{4}^{2}\right) a_{14} \cos \left(\xi_{3}\right)}{a_{4}^{2}}\right]$,

where the functions $F, \xi_{1}, \xi_{2}$ and $\xi_{3}$ are given as follows:

$$
\begin{aligned}
F= & \mathrm{e}^{-a_{4} t-\frac{\left(a_{3}^{2}-a_{4}^{2}\right) x}{a_{4}}-a_{3} z-a_{5}}+k_{1} \mathrm{e}^{a_{4}+\frac{\left(a_{3}^{2}-a_{4}^{2}\right) x}{a_{4}}+a_{3} z+a_{5}} \\
& +k_{2} \cos \left[a_{9} t+\frac{\left(a_{3}^{2}-a_{4}^{2}\right) a_{9} y}{a_{4}^{2}}+\frac{a_{3} a_{9} z}{a_{4}}+a_{10}\right] \\
& +k_{3} \sin \left[a_{14} t+\frac{\left(a_{3}^{2}-a_{4}^{2}\right) a_{14} x}{a_{4}^{2}}+\frac{a_{3} a_{14} z}{a_{4}}+a_{15}\right]+a_{16}, \\
\xi_{1}= & a_{4} t+\frac{\left(a_{3}^{2}-a_{4}^{2}\right) x}{a_{4}}+a_{3} z+a_{5}, \\
\xi_{2}= & a_{9} t+\frac{\left(a_{3}^{2}-a_{4}^{2}\right) a_{9} y}{a_{4}^{2}}+\frac{a_{3} a_{9} z}{a_{4}}+a_{10}, \\
\xi_{3}= & a_{14} t+\frac{\left(a_{3}^{2}-a_{4}^{2}\right) a_{14} x}{a_{4}^{2}}+\frac{a_{3} a_{14} z}{a_{4}}+a_{15} .
\end{aligned}
$$

Case 2:

$$
\begin{aligned}
& a_{1}=\frac{a_{3}^{2}-a_{4}^{2}}{a_{4}}, a_{5}=a_{5}, a_{2}=0, a_{3}=a_{3}, a_{4}=a_{4}, a_{6}=\frac{\left(a_{3}^{2}-a_{4}^{2}\right) a_{9}}{a_{4}^{2}}, a_{7}=0, \\
& a_{8}=\frac{a_{3} a_{9}}{a_{4}}, a_{9}=a_{9}, a_{10}=a_{10}, a_{11}=0, a_{12}=\frac{\left(a_{3}^{2}-a_{4}^{2}\right) a_{14}}{a_{4}^{2}}, a_{13}=\frac{a_{3} a_{14}}{a_{4}}, \\
& a_{14}=a_{14}, a_{15}=a_{15}, a_{16}=a_{16}, k_{1}=k_{1}, k_{2}=k_{2}, k_{3}=k_{3},
\end{aligned}
$$

with the condition $a_{4} \neq 0$. Through the expression (11) and the transformation (2), we get the three-wave solution of the KPB-like equation

$$
u=\frac{2}{F}\left[-\frac{a_{3}^{2}-a_{4}^{2}}{a_{4}} \mathrm{e}^{-a_{4} t-\frac{\left(a_{3}^{2}-a_{4}^{2}\right) x}{a_{4}}-a_{3} z-a_{5}}+\frac{k_{1}\left(a_{3}^{2}-a_{4}^{2}\right) \mathrm{e}^{\xi_{1}}}{a_{4}}-\frac{k_{2}\left(a_{3}^{2}-a_{4}^{2}\right) a_{9} \sin \left(\xi_{2}\right)}{a_{4}^{2}}\right] \text {, }
$$

where the functions $F, \xi_{1}, \xi_{2}$ and $\xi_{3}$ are given as follows:

$$
\begin{aligned}
F= & \mathrm{e}^{-a_{4} t-\frac{\left(a_{3}^{2}-a_{4}^{2}\right) x}{a_{4}}-a_{3} z-a_{5}}+k_{1} \mathrm{e}^{a_{4} t+\frac{\left(a_{3}^{2}-a_{4}^{2}\right) x}{a_{4}}+a_{3} z+a_{5}} \\
& +k_{2} \cos \left[a_{9} t+\frac{\left(a_{3}^{2}-a_{4}^{2}\right) a_{9} x}{a_{4}^{2}}+\frac{a_{3} a_{9} z}{a_{4}}+a_{10}\right] \\
& +k_{3} \sin \left[a_{14} t+\frac{\left(a_{3}^{2}-a_{4}^{2}\right) a_{14} y}{a_{4}^{2}}+\frac{a_{3} a_{14} z}{a_{4}}+a_{15}\right]+a_{16},
\end{aligned}
$$




$$
\begin{aligned}
& \xi_{1}=a_{4} t+\frac{\left(a_{3}^{2}-a_{4}^{2}\right) x}{a_{4}}+a_{3} z+a_{5}, \\
& \xi_{2}=a_{9} t+\frac{\left(a_{3}^{2}-a_{4}^{2}\right) a_{9} x}{a_{4}^{2}}+\frac{a_{3} a_{9} z}{a_{4}}+a_{10}, \\
& \xi_{3}=a_{14} t+\frac{\left(a_{3}^{2}-a_{4}^{2}\right) a_{14} y}{a_{4}^{2}}+\frac{a_{3} a_{14} z}{a_{4}}+a_{15} .
\end{aligned}
$$

\section{Case 3:}

$$
\begin{aligned}
& a_{1}=\frac{a_{3}^{2}-a_{4}^{2}}{a_{4}}, a_{2}=0, a_{3}=a_{3}, a_{4}=a_{4}, a_{5}=a_{5}, a_{6}=0, a_{7}=\frac{\left(a_{3}^{2}-a_{4}^{2}\right) a_{9}}{a_{4}^{2}}, \\
& a_{8}=\frac{a_{3} a_{9}}{a_{4}}, a_{9}=a_{9}, a_{10}=a_{10}, a_{11}=0, a_{12}=\frac{\left(a_{3}^{2}-a_{4}^{2}\right) a_{14}}{a_{4}^{2}}, a_{13}=\frac{a_{3} a_{14}}{a_{4}}, \\
& a_{14}=a_{14}, a_{15}=a_{15}, a_{16}=a_{16}, k_{1}=k_{1}, k_{2}=k_{2}, k_{3}=k_{3},
\end{aligned}
$$

with the condition $a_{4} \neq 0$. Through the expression (11) and the transformation (2), we get the three-wave solution of the KPB-like equation

$$
u=\frac{2}{F}\left[-\frac{a_{3}^{2}-a_{4}^{2}}{a_{4}} \mathrm{e}^{-a_{4} t-\frac{\left(a_{3}^{2}-a_{4}^{2}\right) x}{a_{4}}-a_{3} z-a_{5}}+\frac{k_{1}\left(a_{3}^{2}-a_{4}^{2}\right) \mathrm{e}^{\xi_{1}}}{a_{4}}\right],
$$

where the functions $F, \xi_{1}, \xi_{2}$ and $\xi_{3}$ are given as follows:

$$
\begin{aligned}
F= & \mathrm{e}^{-a_{4} t-\frac{\left(a_{3}^{2}-a_{4}^{2}\right) x}{a_{4}}-a_{3} z-a_{5}}+k_{1} \mathrm{e}^{a_{4} t+\frac{\left(a_{3}^{2}-a_{4}^{2}\right) x}{a_{4}}+a_{3} z+a_{5}} \\
& +k_{2} \cos \left[a_{9} t+\frac{\left(a_{3}^{2}-a_{4}^{2}\right) a_{9} y}{a_{4}^{2}}+\frac{a_{3} a_{9} z}{a_{4}}+a_{10}\right] \\
& +k_{3} \sin \left[a_{14} t+\frac{\left(a_{3}^{2}-a_{4}^{2}\right) a_{14} y}{a_{4}^{2}}+\frac{a_{3} a_{14} z}{a_{4}}+a_{15}\right]+a_{16}, \\
\xi_{1}= & a_{4} t+\frac{\left(a_{3}^{2}-a_{4}^{2}\right) x}{a_{4}}+a_{3} z+a_{5}, \\
\xi_{2}= & a_{9} t+\frac{\left(a_{3}^{2}-a_{4}^{2}\right) a_{9} y}{a_{4}^{2}}+\frac{a_{3} a_{9} z}{a_{4}}+a_{10}, \\
\xi_{3}= & a_{14} t+\frac{\left(a_{3}^{2}-a_{4}^{2}\right) a_{14} y}{a_{4}^{2}}+\frac{a_{3} a_{14} z}{a_{4}}+a_{15} .
\end{aligned}
$$

\section{Case 4:}

$$
\begin{aligned}
& a_{1}=0, a_{2}=\frac{a_{3}^{2}-a_{4}^{2}}{a_{4}}, a_{3}=a_{3}, a_{4}=a_{4}, a_{5}=a_{5}, a_{6}=0, a_{7}=\frac{\left(a_{3}^{2}-a_{4}^{2}\right) a_{9}}{a_{4}^{2}}, \\
& a_{8}=\frac{a_{3} a_{9}}{a_{4}}, a_{9}=a_{9}, a_{10}=a_{10}, a_{11}=\frac{\left(a_{3}^{2}-a_{4}^{2}\right) a_{14}}{a_{4}^{2}}, a_{12}=0, a_{13}=\frac{a_{3} a_{14}}{a_{4}}, \\
& a_{14}=a_{14}, a_{15}=a_{15}, a_{16}=a_{16}, k_{1}=k_{1}, k_{2}=k_{2}, k_{3}=k_{3},
\end{aligned}
$$


with the condition $a_{4} \neq 0$. Through the expression (11) and the transformation (2), we get the three-wave solution of the KPB-like equation

$$
u=\frac{2 k_{3}\left(a_{3}^{2}-a_{4}^{2}\right) a_{14} \cos \left(\xi_{3}\right)}{a_{4}^{2} F},
$$

where the functions $F, \xi_{1}, \xi_{2}$ and $\xi_{3}$ are given as follows:

$$
\begin{aligned}
F= & \mathrm{e}^{-a_{4} t-\frac{\left(a_{3}^{2}-a_{4}^{2}\right) y}{a_{4}}-a_{3} z-a_{5}}+k_{1} \mathrm{e}^{a_{4} t+\frac{\left(a_{3}^{2}-a_{4}^{2}\right) y}{a_{4}}+a_{3} z+a_{5}} \\
& +k_{2} \cos \left[a_{9} t+\frac{\left(a_{3}^{2}-a_{4}^{2}\right) a_{9} y}{a_{4}^{2}}+\frac{a_{3} a_{9} z}{a_{4}}+a_{10}\right] \\
& +k_{3} \sin \left[a_{14} t+\frac{\left(a_{3}^{2}-a_{4}^{2}\right) a_{14} x}{a_{4}^{2}}+\frac{a_{3} a_{14} z}{a_{4}}+a_{15}\right]+a_{16}, \\
\xi_{1}= & a_{4} t+\frac{\left(a_{3}^{2}-a_{4}^{2}\right) y}{a_{4}}+a_{3} z+a_{5}, \\
\xi_{2}= & a_{9} t+\frac{\left(a_{3}^{2}-a_{4}^{2}\right) a_{9} y}{a_{4}^{2}}+\frac{a_{3} a_{9} z}{a_{4}}+a_{10}, \\
\xi_{3}= & a_{14} t+\frac{\left(a_{3}^{2}-a_{4}^{2}\right) a_{14} x}{a_{4}^{2}}+\frac{a_{3} a_{14} z}{a_{4}}+a_{15} .
\end{aligned}
$$

Case 5:

$$
\begin{aligned}
& a_{1}=0, a_{2}=\frac{a_{3}^{2}-a_{4}^{2}}{a_{4}}, a_{3}=a_{3}, a_{4}=a_{4}, a_{5}=a_{5}, \\
& a_{6}=\frac{\left(a_{3}^{2}-a_{4}^{2}\right) a_{9}}{a_{4}^{2}}, a_{7}=0, a_{8}=\frac{a_{3} a_{9}}{a_{4}}, a_{9}=a_{9}, \\
& a_{10}=a_{10}, a_{11}=0, a_{12}=\frac{\left(a_{3}^{2}-a_{4}^{2}\right) a_{14}}{a_{4}^{2}}, a_{13}=\frac{a_{3} a_{14}}{a_{4}}, \\
& a_{14}=a_{14}, a_{15}=a_{15}, a_{16}=a_{16}, k_{1}=k_{1}, k_{2}=k_{2}, k_{3}=k_{3},
\end{aligned}
$$

with the condition $a_{4} \neq 0$. Through the expression (11) and the transformation (2), we get the three-wave solution of the KPB-like equation

$$
u=\frac{-2 k_{2} a_{9}\left(a_{3}^{2}-a_{4}^{2}\right) \sin \left(\xi_{2}\right)}{a_{4}^{2} F},
$$

where the functions $F, \xi_{1}, \xi_{2}$ and $\xi_{3}$ are given as follows:

$$
\begin{aligned}
F= & \mathrm{e}^{-a_{4} t-\frac{\left(a_{3}^{2}-a_{4}^{2}\right) y}{a_{4}}-a_{3} z-a_{5}}+k_{1} \mathrm{e}^{a_{4} t+\frac{\left(a_{3}^{2}-a_{4}^{2}\right) y}{a_{4}}+a_{3} z+a_{5}} \\
& +k_{2} \cos \left[a_{9} t+\frac{\left(a_{3}^{2}-a_{4}^{2}\right) a_{9} x}{a_{4}^{2}}+\frac{a_{3} a_{9} z}{a_{4}}+a_{10}\right] \\
& +k_{3} \sin \left[a_{14} t+\frac{\left(a_{3}^{2}-a_{4}^{2}\right) a_{14} y}{a_{4}^{2}}+\frac{a_{3} a_{14} z}{a_{4}}+a_{15}\right]+a_{16},
\end{aligned}
$$




$$
\begin{aligned}
& \xi_{1}=a_{4} t+\frac{\left(a_{3}^{2}-a_{4}^{2}\right) y}{a_{4}}+a_{3} z+a_{5}, \\
& \xi_{2}=a_{9} t+\frac{\left(a_{3}^{2}-a_{4}^{2}\right) a_{9} x}{a_{4}^{2}}+\frac{a_{3} a_{9} z}{a_{4}}+a_{10}, \\
& \xi_{3}=a_{14} t+\frac{\left(a_{3}^{2}-a_{4}^{2}\right) a_{14} y}{a_{4}^{2}}+\frac{a_{3} a_{14} z}{a_{4}}+a_{15} .
\end{aligned}
$$

\section{Case 6:}

$$
\begin{aligned}
& a_{1}=0, a_{2}=\frac{a_{3}^{2}-a_{4}^{2}}{a_{4}}, a_{3}=a_{3}, a_{4}=a_{4}, a_{5}=a_{5}, a_{6}=\frac{\left(a_{3}^{2}-a_{4}^{2}\right) a_{9}}{a_{4}^{2}}, a_{7}=0, \\
& a_{8}=\frac{a_{3} a_{9}}{a_{4}}, a_{9}=a_{9}, a_{10}=a_{10}, a_{11}=\frac{\left(a_{3}^{2}-a_{4}^{2}\right) a_{14}}{a_{4}^{2}}, a_{12}=0, a_{13}=\frac{a_{3} a_{14}}{a_{4}}, \\
& a_{14}=a_{14}, a_{15}=a_{15}, a_{16}=a_{16}, k_{1}=k_{1}, k_{2}=k_{2}, k_{3}=k_{3},
\end{aligned}
$$

with the condition $a_{4} \neq 0$. Through the expression (11) and the transformation (2), we get the three-wave solution of the KPB-like equation

$$
u=\frac{2}{F}\left[-\frac{k_{2}\left(a_{3}^{2}-a_{4}^{2}\right) a_{9} \sin \left(\xi_{2}\right)}{a_{4}^{2}}+\frac{k_{3}\left(a_{3}^{2}-a_{4}^{2}\right) a_{14} \cos \left(\xi_{3}\right)}{a_{4}^{2}}\right],
$$

where the functions $F, \xi_{1}, \xi_{2}$ and $\xi_{3}$ are given as follows:

$$
\begin{aligned}
F= & \mathrm{e}^{-a_{4} t-\frac{\left(a_{3}^{2}-a_{4}^{2}\right) y}{a_{4}}-a_{3} z-a_{5}}+k_{1} \mathrm{e}^{a_{4} t+\frac{\left(a_{3}^{2}-a_{4}^{2}\right) y}{a_{4}}+a_{3} z+a_{5}} \\
& +k_{2} \cos \left[a_{9} t+\frac{\left(a_{3}^{2}-a_{4}^{2}\right) a_{9} x}{a_{4}^{2}}+\frac{a_{3} a_{9} z}{a_{4}}+a_{10}\right] \\
& +k_{3} \sin \left[a_{14} t+\frac{\left(a_{3}^{2}-a_{4}^{2}\right) a_{14} x}{a_{4}^{2}}+\frac{a_{3} a_{14} z}{a_{4}}+a_{15}\right]+a_{16}, \\
\xi_{1}= & a_{4} t+\frac{\left(a_{3}^{2}-a_{4}^{2}\right) y}{a_{4}}+a_{3} z+a_{5}, \\
\xi_{2}= & a_{9} t+\frac{\left(a_{3}^{2}-a_{4}^{2}\right) a_{9} x}{a_{4}^{2}}+\frac{a_{3} a_{9} z}{a_{4}}+a_{10}, \\
\xi_{3}= & a_{14} t+\frac{\left(a_{3}^{2}-a_{4}^{2}\right) a_{14} x}{a_{4}^{2}}+\frac{a_{3} a_{14} z}{a_{4}}+a_{15} .
\end{aligned}
$$

In order to analyze the dynamics properties briefly, we would like to discuss the evolution characteristic. By choosing appropriate values of these parameters in (13), we set

$$
\begin{aligned}
& Z=x, a_{3}=1, a_{4}=2, a_{5}=2, a_{9}=2, a_{10}=2, a_{14}=2, \\
& a_{15}=2, a_{16}=2, k_{1}=1, k_{2}=1, k_{3}=1 .
\end{aligned}
$$

The three-dimensional dynamic graphs of the wave and corresponding density plots and contour plots were successfully depicted in Figure 1 with the help of Maple. We can see the exponential function wave and the sine-cosine function wave of KPB-like equation. 

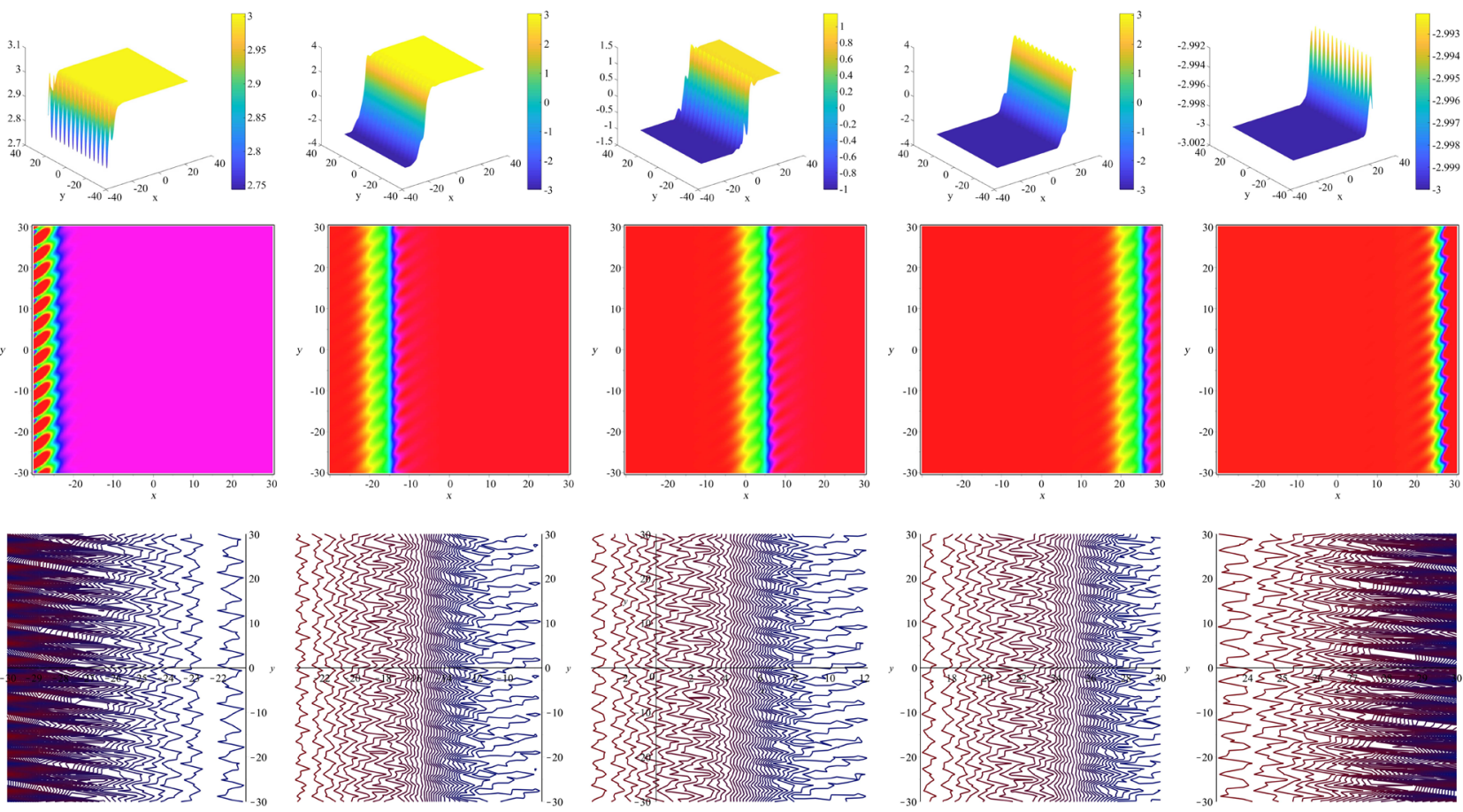

(a) $\mathrm{t}=-10$

(b) $t=-5$

(c) $\mathrm{t}=0$

(d) $\mathrm{t}=5$

(e) $\mathrm{t}=10$

Figure 1. Evolution plots (top), density plot (middle) and contour plots (bottom) of the Equation (13) by choosing $Z=x$, $a_{3}=1, a_{4}=2, a_{5}=2, a_{9}=2, a_{10}=2, a_{14}=2, a_{15}=2, a_{16}=2, k_{1}=1, k_{2}=1, k_{3}=1$.

\section{The New Periodic Solitary Wave Solutions of the KPB-Like Equation}

To search for the new periodic solitary wave solutions of the KPB-like Equation (1), we suppose [11]

$$
\begin{aligned}
& f=\mathrm{e}^{-G}+k_{1} \mathrm{e}^{G}+k_{2} \tan (H)+k_{3} \tanh (N)+a_{16}, \\
& G=a_{1} x+a_{2} y+a_{3} z+a_{4} t+a_{5}, \\
& H=a_{6} x+a_{7} y+a_{8} z+a_{9} t+a_{10}, \\
& N=a_{11} x+a_{12} y+a_{13} z+a_{14} t+a_{15},
\end{aligned}
$$

where $a_{i}(i=1,2, \cdots, 16)$ and $k_{i}(i=1,2,3)$ are real parameters to be determined later. With the help of Maple, substituting (31) into Equation (3), we obtain a set of algebraic equations in $a_{i}(i=1,2, \cdots, 16)$ and $k_{i}(i=1,2,3)$. Solving the set of algebraic equations, we can find the following sets of solutions and these set leads to the corresponding periodic solitary wave solutions of the KPB-like equation.

\section{Case 1:}

$$
\begin{aligned}
& a_{1}=\frac{a_{3}^{2}-a_{4}^{2}}{a_{4}}, a_{2}=0, a_{3}=a_{3}, a_{4}=a_{4}, a_{5}=a_{5}, a_{6}=\frac{\left(a_{3}^{2}-a_{4}^{2}\right) a_{9}}{a_{4}^{2}}, a_{7}=0, \\
& a_{8}=\frac{a_{3} a_{9}}{a_{4}}, a_{9}=a_{9}, a_{10}=a_{10}, a_{11}=0, a_{12}=\frac{\left(a_{3}^{2}-a_{4}^{2}\right) a_{14}}{a_{4}^{2}}, a_{13}=\frac{a_{3} a_{14}}{a_{4}}, \\
& a_{14}=a_{14}, a_{15}=a_{15}, a_{16}=0, k_{1}=k_{1}, k_{2}=k_{2}, k_{3}=k_{3},
\end{aligned}
$$


with the condition $a_{4} \neq 0$.

Case 2:

$$
\begin{aligned}
& a_{1}=\frac{a_{3}^{2}-a_{4}^{2}}{a_{4}}, a_{2}=0, a_{3}=a_{3}, a_{4}=a_{4}, a_{5}=a_{5}, a_{6}=0, a_{7}=\frac{\left(a_{3}^{2}-a_{4}^{2}\right) a_{9}}{a_{4}^{2}}, \\
& a_{8}=\frac{a_{3} a_{9}}{a_{4}}, a_{9}=a_{9}, a_{10}=a_{10}, a_{11}=\frac{\left(a_{3}^{2}-a_{4}^{2}\right) a_{14}}{a_{4}^{2}}, a_{12}=0, a_{13}=\frac{a_{3} a_{14}}{a_{4}}, \\
& a_{14}=a_{14}, a_{15}=a_{15}, a_{16}=0, k_{1}=k_{1}, k_{2}=k_{2}, k_{3}=k_{3},
\end{aligned}
$$

with the condition $a_{4} \neq 0$.

Case 3:

$$
\begin{aligned}
& a_{1}=\frac{a_{3}^{2}-a_{4}^{2}}{a_{4}}, a_{2}=0, a_{3}=a_{3}, a_{4}=a_{4}, a_{5}=a_{5}, a_{6}=0, a_{7}=\frac{\left(a_{3}^{2}-a_{4}^{2}\right) a_{9}}{a_{4}^{2}}, \\
& a_{8}=\frac{a_{3} a_{9}}{a_{4}}, a_{9}=a_{9}, a_{10}=a_{10}, a_{11}=0, a_{12}=\frac{\left(a_{3}^{2}-a_{4}^{2}\right) a_{14}}{a_{4}^{2}}, a_{13}=\frac{a_{3} a_{14}}{a_{4}}, \\
& a_{14}=a_{14}, a_{15}=a_{15}, a_{16}=0, k_{1}=k_{1}, k_{2}=k_{2}, k_{3}=k_{3},
\end{aligned}
$$

with the condition $a_{4} \neq 0$.

Case 4:

$$
\begin{aligned}
& a_{1}=0, a_{2}=\frac{a_{3}^{2}-a_{4}^{2}}{a_{4}}, a_{3}=a_{3}, a_{4}=a_{4}, a_{5}=a_{5}, a_{6}=\frac{\left(a_{3}^{2}-a_{4}^{2}\right) a_{9}}{a_{4}^{2}}, a_{7}=0, \\
& a_{8}=\frac{a_{3} a_{9}}{a_{4}}, a_{9}=a_{9}, a_{10}=a_{10}, a_{11}=\frac{\left(a_{3}^{2}-a_{4}^{2}\right) a_{14}}{a_{4}^{2}}, a_{12}=0, a_{13}=\frac{a_{3} a_{14}}{a_{4}}, \\
& a_{14}=a_{14}, a_{15}=a_{15}, a_{16}=0, k_{1}=k_{1}, k_{2}=k_{2}, k_{3}=k_{3},
\end{aligned}
$$

with the condition $a_{4} \neq 0$.

Case 5:

$$
\begin{aligned}
& a_{1}=0, a_{2}=\frac{a_{3}^{2}-a_{4}^{2}}{a_{4}}, a_{3}=a_{3}, a_{4}=a_{4}, a_{5}=a_{5}, a_{6}=\frac{\left(a_{3}^{2}-a_{4}^{2}\right) a_{9}}{a_{4}^{2}}, a_{7}=0, \\
& a_{8}=\frac{a_{3} a_{9}}{a_{4}}, a_{9}=a_{9}, a_{10}=a_{10}, a_{11}=0, a_{12}=\frac{\left(a_{3}^{2}-a_{4}^{2}\right) a_{14}}{a_{4}^{2}}, a_{13}=\frac{a_{3} a_{14}}{a_{4}}, \\
& a_{14}=a_{14}, a_{15}=a_{15}, a_{16}=0, k_{1}=k_{1}, k_{2}=k_{2}, k_{3}=k_{3},
\end{aligned}
$$

with the condition $a_{4} \neq 0$.

Case 6:

$$
\begin{aligned}
& a_{1}=0, a_{2}=\frac{a_{3}^{2}-a_{4}^{2}}{a_{4}}, a_{3}=a_{3}, a_{4}=a_{4}, a_{5}=a_{5}, a_{6}=0, a_{7}=\frac{\left(a_{3}^{2}-a_{4}^{2}\right) a_{9}}{a_{4}^{2}}, \\
& a_{8}=\frac{a_{3} a_{9}}{a_{4}}, a_{9}=a_{9}, a_{10}=a_{10}, a_{11}=\frac{\left(a_{3}^{2}-a_{4}^{2}\right) a_{14}}{a_{4}^{2}}, a_{12}=0, a_{13}=\frac{a_{3} a_{14}}{a_{4}}, \\
& a_{14}=a_{14}, a_{15}=a_{15}, a_{16}=0, k_{1}=k_{1}, k_{2}=k_{2}, k_{3}=k_{3},
\end{aligned}
$$

with the condition $a_{4} \neq 0$.

Case 7: 


$$
\begin{aligned}
& a_{1}=\frac{a_{4}\left(a_{8}^{2}-a_{9}^{2}\right)}{a_{9}^{2}}, a_{2}=0, a_{3}=\frac{a_{4} a_{8}}{a_{9}}, a_{4}=a_{4}, a_{5}=a_{5}, a_{6}=\frac{a_{8}^{2}-a_{9}^{2}}{a_{9}}, \\
& a_{7}=0, a_{8}=a_{8}, a_{9}=a_{9}, a_{10}=a_{10}, a_{11}=0, a_{12}=\frac{\left(a_{8}^{2}-a_{9}^{2}\right) a_{14}}{a_{9}^{2}}, \\
& a_{13}=\frac{a_{8} a_{14}}{a_{9}}, a_{14}=a_{14}, a_{16}=a_{16}, k_{1}=k_{1}, k_{2}=k_{2}, k_{3}=k_{3},
\end{aligned}
$$

with the condition $a_{9} \neq 0$.

Case 8:

$$
\begin{aligned}
& a_{1}=\frac{a_{4}\left(a_{8}^{2}-a_{9}^{2}\right)}{a_{9}^{2}}, a_{2}=0, a_{3}=\frac{a_{4} a_{8}}{a_{9}}, a_{4}=a_{4}, a_{5}=a_{5}, a_{6}=0, a_{7}=\frac{a_{8}^{2}-a_{9}^{2}}{a_{9}}, \\
& a_{8}=a_{8}, a_{9}=a_{9}, a_{10}=a_{10}, a_{11}=\frac{\left(a_{8}^{2}-a_{9}^{2}\right) a_{14}}{a_{9}^{2}}, a_{12}=0, a_{13}=\frac{a_{8} a_{14}}{a_{9}}, \\
& a_{14}=a_{14}, a_{15}=a_{15}, a_{16}=a_{16}, k_{1}=k_{1}, k_{2}=k_{2}, k_{3}=k_{3},
\end{aligned}
$$

with the condition $a_{9} \neq 0$.

Case 9:

$$
\begin{aligned}
& a_{1}=\frac{a_{4}\left(a_{8}^{2}-a_{9}^{2}\right)}{a_{9}^{2}}, a_{2}=0, a_{3}=\frac{a_{4} a_{8}}{a_{9}}, a_{4}=a_{4}, a_{5}=a_{5}, a_{6}=0, a_{7}=\frac{a_{8}^{2}-a_{9}^{2}}{a_{9}}, \\
& a_{8}=a_{8}, a_{9}=a_{9}, a_{10}=a_{10}, a_{11}=0, a_{12}=\frac{\left(a_{8}^{2}-a_{9}^{2}\right) a_{14}}{a_{9}^{2}}, a_{13}=\frac{a_{8} a_{14}}{a_{9}}, \\
& a_{14}=a_{14}, a_{15}=a_{15}, a_{16}=a_{16}, k_{1}=k_{1}, k_{2}=k_{2}, k_{3}=k_{3},
\end{aligned}
$$

with the condition $a_{9} \neq 0$.

Case 10:

$$
\begin{aligned}
& a_{1}=0, a_{2}=\frac{a_{4}\left(a_{8}^{2}-a_{9}^{2}\right)}{a_{9}^{2}}, a_{3}=\frac{a_{4} a_{8}}{a_{9}}, a_{4}=a_{4}, a_{5}=a_{5}, a_{6}=\frac{a_{8}^{2}-a_{9}^{2}}{a_{9}}, a_{7}=0, \\
& a_{8}=a_{8}, a_{9}=a_{9}, a_{10}=a_{10}, a_{11}=\frac{\left(a_{8}^{2}-a_{9}^{2}\right) a_{14}}{a_{9}^{2}}, a_{12}=0, a_{13}=\frac{a_{8} a_{14}}{a_{9}}, \\
& a_{14}=a_{14}, a_{15}=a_{15}, a_{16}=a_{16}, k_{1}=k_{1}, k_{2}=k_{2}, k_{3}=k_{3},
\end{aligned}
$$

with the condition $a_{9} \neq 0$.

Case 11:

$$
\begin{aligned}
& a_{1}=0, a_{2}=\frac{a_{4}\left(a_{8}^{2}-a_{9}^{2}\right)}{a_{9}^{2}}, a_{3}=\frac{a_{4} a_{8}}{a_{9}}, a_{4}=a_{4}, a_{5}=a_{5}, a_{6}=\frac{a_{8}^{2}-a_{9}^{2}}{a_{9}}, a_{7}=0, \\
& a_{8}=a_{8}, a_{9}=a_{9}, a_{10}=a_{10}, a_{11}=0, a_{12}=\frac{\left(a_{8}^{2}-a_{9}^{2}\right) a_{14}}{a_{9}^{2}}, a_{13}=\frac{a_{8} a_{14}}{a_{9}}, \\
& a_{14}=a_{14}, a_{15}=a_{15}, a_{16}=a_{16}, k_{1}=k_{1}, k_{2}=k_{2}, k_{3}=k_{3},
\end{aligned}
$$

with the condition $a_{9} \neq 0$.

Case 12: 


$$
\begin{aligned}
& a_{1}=0, a_{2}=\frac{a_{4}\left(a_{8}^{2}-a_{9}^{2}\right)}{a_{9}^{2}}, a_{3}=\frac{a_{4} a_{8}}{a_{9}}, a_{4}=a_{4}, a_{5}=a_{5}, a_{6}=0, a_{7}=\frac{a_{8}^{2}-a_{9}^{2}}{a_{9}}, \\
& a_{8}=a_{8}, a_{9}=a_{9}, a_{10}=a_{10}, a_{11}=\frac{\left(a_{8}^{2}-a_{9}^{2}\right) a_{14}}{a_{9}^{2}}, a_{12}=0, a_{13}=\frac{a_{8} a_{14}}{a_{9}}, \\
& a_{14}=a_{14}, a_{15}=a_{15}, a_{16}=a_{16}, k_{1}=k_{1}, k_{2}=k_{2}, k_{3}=k_{3},
\end{aligned}
$$

with the condition $a_{9} \neq 0$.

To search the periodic solution of the KPB-like equation, for example, let's try to substitute the solution (32) into the expression (31). Then through the expression (31) and the transformation (2), we get the periodic solitary wave solution of the KPB-like equation,

$$
\begin{aligned}
u= & \frac{2}{f}\left[-\frac{a_{3}^{2}-a_{4}^{2}}{a_{4}} \mathrm{e}^{-a_{4} t-\frac{\left(a_{3}^{2}-a_{4}^{2}\right) x}{a_{4}}-a_{3} z-a_{5}}+\frac{k_{1}\left(a_{3}^{2}-a_{4}^{2}\right) \mathrm{e}^{\xi_{1}}}{a_{4}}\right. \\
& \left.+\frac{k_{2}\left(a_{3}^{2}-a_{4}^{2}\right) a_{9}\left(1+\left(\tan \left(\xi_{2}\right)\right)^{2}\right)}{a_{4}^{2}}\right]
\end{aligned}
$$

where the functions $F, \xi_{1}, \xi_{2}$ and $\xi_{3}$ are given as follows:

$$
\begin{aligned}
f= & \mathrm{e}^{-a_{4} t-\frac{\left(a_{3}^{2}-a_{4}^{2}\right) x}{a_{4}}-a_{3} z-a_{5}}+k_{1} \mathrm{e}^{a_{4} t+\frac{\left(a_{3}^{2}-a_{4}^{2}\right) x}{a_{4}}+a_{3} z+a_{5}} \\
& +k_{2} \tan \left[a_{9} t+\frac{\left(a_{3}^{2}-a_{4}^{2}\right) a_{9} x}{a_{4}^{2}}+\frac{a_{3} a_{9} z}{a_{4}}+a_{10}\right] \\
& +k_{3} \tanh \left[a_{14} t+\frac{\left(a_{3}^{2}-a_{4}^{2}\right) a_{14} y}{a_{4}^{2}}+\frac{a_{3} a_{14} z}{a_{4}}+a_{15}\right]+a_{16}, \\
\xi_{1}= & a_{4} t+\frac{\left(a_{3}^{2}-a_{4}^{2}\right) x}{a_{4}}+a_{3} z+a_{5}, \\
\xi_{2}= & a_{9} t+\frac{\left(a_{3}^{2}-a_{4}^{2}\right) a_{9} x}{a_{4}^{2}}+\frac{a_{3} a_{9} z}{a_{4}}+a_{10}, \\
\xi_{3}= & a_{14} t+\frac{\left(a_{3}^{2}-a_{4}^{2}\right) a_{14} y}{a_{4}^{2}}+\frac{a_{3} a_{14} z}{a_{4}}+a_{15} .
\end{aligned}
$$

In order to analyze the dynamics properties briefly, we would like to discuss the evolution characteristic. By choosing appropriate values of these parameters in (44), we set

$$
\begin{aligned}
& z=y, a_{3}=1, a_{4}=2, a_{5}=2, a_{9}=2, a_{10}=2, a_{14}=2, \\
& a_{15}=2, a_{16}=2, k_{1}=1, k_{2}=1, k_{3}=1 .
\end{aligned}
$$

The three-dimensional dynamic graphs of the wave and corresponding density plots and contour plots were successfully depicted in Figure 2 with the help of Maple. In Figure 2, we can see that when $t=-10$ to $t=10$, the wave is generated and disappeared, which is the result of the interaction of exponential function wave, tangent function wave and hyperbolic tangent function wave. 


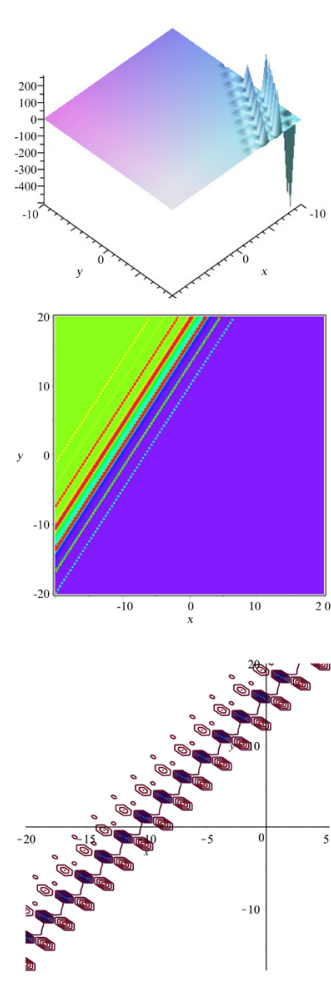

(a) $t=-10$
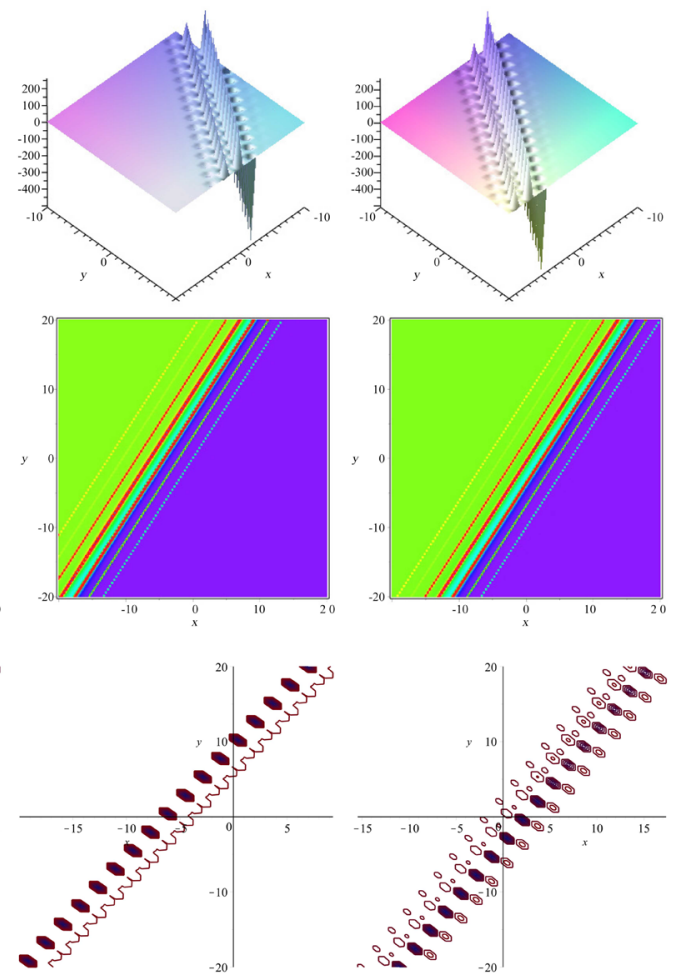

(b) $t=-5$

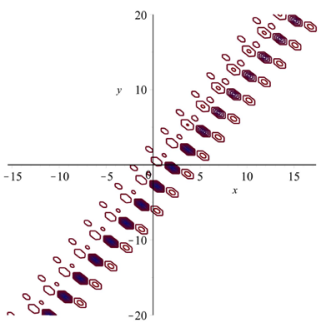

(c) $\mathrm{t}=0$
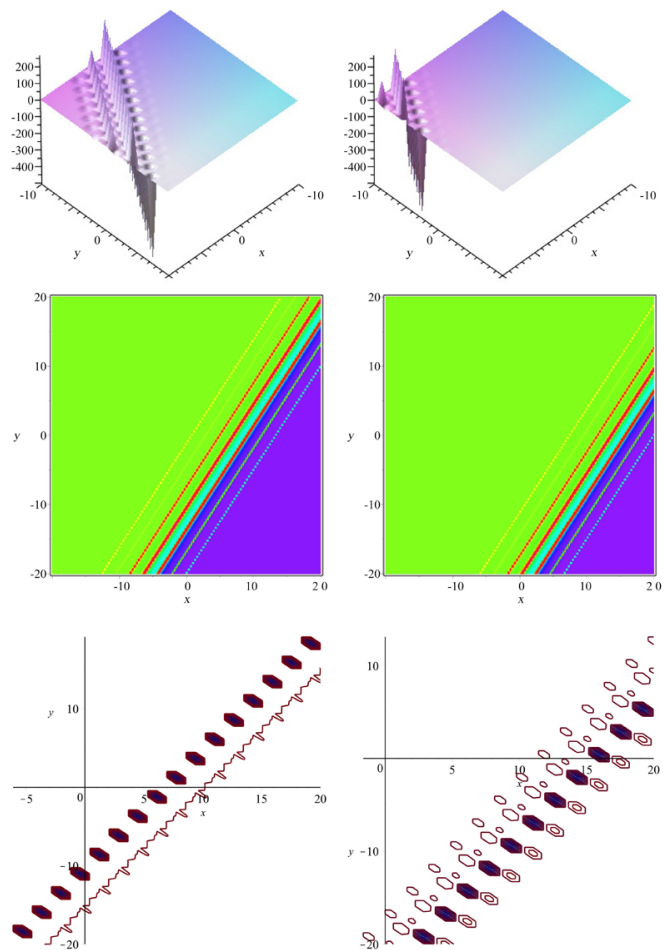

(d) $\mathrm{t}=5$

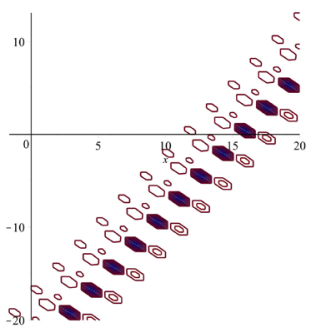

(e) $\mathrm{t}=10$

Figure 2. Evolution plots (top), density plot (middle) and contour plots (bottom) of the Equation (44) by choosing $z=y$, $a_{3}=1, a_{4}=2, a_{5}=2, a_{9}=2, a_{10}=2, a_{14}=2, a_{15}=2, a_{16}=2, k_{1}=1, k_{2}=1, k_{3}=1$.

\section{Conclusions and Outlook}

In this paper, with the help of Maple, we obtained new three-wave solutions and new periodic solitary wave solutions of the $(3+1)$-dimensional KPB-like equation, and successfully depicted the three-dimensional evolution map of the wave and the corresponding density map of the new three-wave solutions and new periodic solitory wave solution, from which we can see the exponential function wave. The new three-wave solutions and new periodic solitary wave solutions obtained in this paper, will have a wide range of applications in the fields of physics and mechanics.

In fact, we can further calculate other exact solutions of the KPB-like equation and other NLEEs; when $p=5$ and $p=7$ by generalized bilinear derivative, the new three-wave solutions and new periodic solitary wave solutions of those two classes of equations will be obtained with different calculation.

\section{Acknowledgements}

This work is supported by the National Natural Science Foundation of China (11661060, 12061054), the Natural Science Foundation of Inner Mongolia Autonomous Region of China (2018LH01013) and Program for Young Talents of Science and Technology in Universities of Inner Mongolia Autonomous Region (NJYT-20-A06). 


\section{Conflicts of Interest}

The authors declare no conflicts of interest regarding the publication of this paper.

\section{References}

[1] Ablowitz, M.J. and Clarkson, P.A. (1991) Solitons, Nonlinear Evolution Equations and Inverse Scattering. Cambridge University Press, Cambridge. https://doi.org/10.1017/CBO9780511623998

[2] Wazwaz, A.M. (2017) Two-Mode Fifth-Order KDV Equations: Necessary Conditions for Multiple-Soliton Solutions to Exist. Nonlinear Dynamics, 87, 1685-1691. https://doi.org/10.1007/s11071-016-3144-Z

[3] Yang, B. and Chen, Y. (2018) Dynamics of High-Order Solitons in the Nonlocal Nonlinear Schrödinger Equations. Nonlinear Dynamics, 94, 489-502. https://doi.org/10.1007/s11071-018-4373-0

[4] Lü, X. and Lin, F.H. (2016) Soliton Excitations and Shape-Changing Collisions in Alpha Helical Proteins with Interspine Coupling at Higher Order. Communications in Nonlinear Science and Numerical Simulation, 32, 241-261.

https://doi.org/10.1016/j.cnsns.2015.08.008

[5] Bilige, S.D., Chaolu, T. and Wang, X.M. (2013) Application of the Extend Simplest Equation Method to the Coupled Schrödinger-Boussinesq Equation. Applied Mathematics and Computation, 224, 517-523. https://doi.org/10.1016/j.amc.2013.08.083

[6] Roshid, H.O. (2019) Multi-Soliton of the (2+1)-Dimensional Calogero-BogoyavlenskiiSchiff Equation and KdV Equation. Computational Methods for Differential Equations, 7, 86-95.

[7] Zhang, X.E. and Chen, Y. (2018) General High-Order Rogue Waves to Nonlinear Schrödinger-Boussinesq Equation with the Dynamical Analysis. Nonlinear Dynamics, 93, 2169-2184. https://doi.org/10.1007/s11071-018-4317-8

[8] Wang, X.B., Tian, S.F., Qin, C.Y. and Zhang, T.T. (2017) Characteristics of the Solitary Waves and Rogue Waves with Interaction Phenomena in a Generalized (3+ 1)-Dimensional Kadomtsev-Petviashvili Equation. Applied Mathematics Letters, 72, 58-64. https://doi.org/10.1016/j.aml.2017.04.009

[9] Lü, Z.S. and Chen, Y.N. (2015) Constructing Rogue Wave Prototypes of Nonlinear Evolution Equations via an Extended Tanh Method. Chaos Solitons and Fractals, 81, 218-223. https://doi.org/10.1016/j.chaos.2015.09.022

[10] Dong, H.H. and Zhang, Y.F. (2015) Exact Periodic Wave Solution of Extended (2+ 1)-Dimensional Shallow Water Wave Equation with Generalized dp Operator. Communications in Theoretical Physics, 63, 401. https://doi.org/10.1088/0253-6102/63/4/401

[11] Liu, J.G. (2018) New Periodic Solitary Wave Solutions for the New (2+1)-Dimensional Kortewegde Vries Equation. Nonlinear Dynamics, 91, 497-504. https://doi.org/10.1007/s11071-017-3884-4

[12] Johnson, R.S. (2009) Periodic Waves over Constant Vorticity: Some Asymptotic Results Generated by Parameter Expansions. Wave Motion, 46, 339-349. https://doi.org/10.1016/j.wavemoti.2009.06.006

[13] Eslami, M. and Mirzazadeh, M. (2016) Optical Solitons with Biswas-Milovic Equation for Power Law and Dual-Power Law Nonlinearities. Nonlinear Dynamics, 83, 1-8. https://doi.org/10.1007/s11071-015-2361-1 
[14] Ma, W.X. (2015) Lump Solutions to the Kadomtsev-Petviashvili Equation. Physics Letters A, 36, 1975-1978. https://doi.org/10.1016/j.physleta.2015.06.061

[15] Ma, W.X. and Zhou, Y. (2018) Lump Solutions to Nonlinear Partial Differential Equations via Hirota Bilinear Forms. Journal of Differential Equations, 264, 2633-2659. https://doi.org/10.1016/j.jde.2017.10.033

[16] Lü, Z.S. and Chen, Y.N. (2015) Construction of Rogue Wave and Lump Solutions for Nonlinear Evolution Equations. The European Physical Journal B, 88, 88-187. https://doi.org/10.1140/epjb/e2015-60099-0

[17] Zhao, Z.L., Chen, Y. and Han, B. (2017) Lump Soliton, Mixed Lump Stripe and Periodic Lump Solutions of a $(2+1)$-Dimensional Asymmetrical Nizhnik Novikov Veselov Equation. Modern Physics Letters B, 31, Article ID: 1750157. https://doi.org/10.1142/S0217984917501573

[18] Hu, W.C., Huang, W.H., Lu, Z.M. and Stepanyants, Y. (2018) Interaction of Multi-Lumps within the Kadomtsev-Petviashvili Equation. Wave Motion, 77, 243-256. https://doi.org/10.1016/j.wavemoti.2017.12.004

[19] Zhang, J.B. and Ma, W.X. (2017) Mixed Lump-Kink Solutions to the BKP Equation. Computers \& Mathematics with Applications, 74, 591-596.

https://doi.org/10.1016/j.camwa.2017.05.010

[20] Foroutan, M., Manafian, J. and Ranjbaran, A. (2018) Lump Solution and Its Interaction to $(3+1)$-D Potential-YTSF Equation. Nonlinear Dynamics, 92, 2077-2092. https://doi.org/10.1007/s11071-018-4182-5

[21] Manafian, J., Foroutan, M. and Ranjbaran, A. (2019) Lump-Type Solutions and Interaction Phenomenon to the Bidirectional Sawada-Kotera Equation. Pramana-Journal of Physics, 92, 41. https://doi.org/10.1007/s12043-018-1700-4

[22] Lü, X. and Ma, W.X. (2016) Study of Lump Dynamics Based on a Dimensionally Reduced Hirota Bilinear Equation. Nonlinear Dynamics, 85, 1217-1222. https://doi.org/10.1007/s11071-016-2755-8

[23] Lü, X., Ma, W.X., Chen, S.T. and Chaudry, M.K. (2016) A Note on Rational Solutions to a Hirota-Satsuma-Like Equation. Applied Mathematics Letters, 58, 13-18. https://doi.org/10.1016/j.aml.2015.12.019

[24] Lü, X., Wang, J.P., Lin, F.H. and Zhou, X.W. (2018) Lump Dynamics of a Generalized Two-Dimensional Boussinesq Equation in Shallowwater. Nonlinear Dynamics, 91, 1249-1259. https://doi.org/10.1007/s11071-017-3942-y

[25] Zhang, X.E. and Chen, Y. (2017) Rogue Wave and a Pair of Resonance Stripe Solitons to a Reduced $(3+1)$-Dimensional Jimbo-Miwa Equation. Communications in Nonlinear Science and Numerical Simulation, 52, 24-31. https://doi.org/10.1016/j.cnsns.2017.03.021

[26] Lü, J.Q., Bilige, S.D. and Chaolu, T. (2018) The Study of Lump Solution and Interaction Phenomenon to $(2+1)$-Dimensional Generalized Fifth-Order Kdv Equation. Nonlinear Dynamics, 91, 1669-1676.

https://doi.org/10.1007/s11071-017-3972-5

[27] Lv, J.Q. and Bilige, S.D. (2017) Lump Solutions of a (2+1)-Dimensional bSK Equation. Nonlinear Dynamics, 90, 2119-2124. https://doi.org/10.1007/s11071-017-3788-3

[28] Lü, J.Q. and Bilige, S.D. (2019) The Study of Lump Solution and Interaction Phenomenon to $(2+1)$-Dimensional Potential Kadomstev-Petviashvili Equation. Analysis and Mathematical Physics, 9, 1497-1509. https://doi.org/10.1007/s13324-018-0256-2

[29] Lü, J.Q., Bilige, S.D. and Gao, X.Q. (2019) Abundant Lump Solution and Interaction 
Phenomenon of $(3+1)$-Dimensional Generalized Kadomtsev-Petviashvili Equation. International Journal of Nonlinear Sciences and Numerical Simulation, 20, 33-40. https://doi.org/10.1515/ijnsns-2018-0034

[30] Zhang, R.F., Bilige, S.D., Bai, Y.X., Lü, J.Q. and Gao, X.Q. (2018) Interaction Phenomenon Todimensionally Reduced p-gBKP Equation. Modern Physics Letters $B$, 32, Article ID: 1850074. https://doi.org/10.1142/S0217984918500744

[31] Lü, J.Q., Bilige, S.D., Gao, X.Q., Bai, Y.X. and Zhang, R.F. (2018) Abundant Lump Solutions and Interaction Phenomena to the Kadomtsev-Petviashvili-BenjaminBona-Mahony Equation. Journal of Applied Mathematics and Physics, 6, 1733-1747. https://doi.org/10.4236/jamp.2018.68148

[32] Zhang, R.F. and Bilige, S.D. (2019) New Interaction Phenomenon and the Periodic Lump Wave for the Jimbo-Miwa Equation. Modern Physics Letters B, 33, Article ID: 1950067. https://doi.org/10.1142/S0217984919500672

[33] Wang, X.M. and Bilige, S.D. (2020) Novel Interaction Phenomena of the $(3+$ 1)-Dimensional Jimbo-Miwa Equation. Communications in Theoretical Physics, 72 , Article ID: 045001. https://doi.org/10.1088/1572-9494/ab690c

[34] Ma, W.X. (2016) Lump-Type Solutions to the $(3+1)$-Dimensional Jimbo-Miwa Equation. International Journal of Nonlinear Sciences and Numerical Simulation, 17, 355-359. https://doi.org/10.1515/ijnsns-2015-0050

[35] Zhang, R.F., Bilige, S.D., Fang, T. and Chaolu, T. (2019) New Periodic Wave, CrossKink Wave and the Interaction Phenomenon for the Jimbo-Miwa-Like Equation. Computers and Mathematics with Applications, 78, 754-764.

https://doi.org/10.1016/j.camwa.2019.02.035

[36] Yu, J.P. (2017) A Direct Backlund Transformation for a $(3+1)$-Dimensional Kadomtsev-Petviashvili-Boussinesq-Like Equation. Nonlinear Dynamics, 90, 22263-22268. https://doi.org/10.1007/s11071-017-3799-0

[37] Lü, X., Chen, S.T. and Ma, W.X. (2016) Constructing Lump Solutions to a Generalized Kadomtsev-Petviashvili-Boussinesq Equation. Nonlinear Dynamics, 86, 523-534. https://doi.org/10.1007/s11071-016-2905-Z

[38] Lü, J.Q. and Bilige, S.D. (2018) Diversity of Interaction Solutions to the $(3+$ 1)-Dimensional Kadomtsev-Petviashvili-Boussinesq-Like Equation. Modern Physics Letters B, 13, Article ID: 1850311. https://doi.org/10.1142/S0217984918503116

[39] Ma, W.X. (2011) Generalized Bilinear Differential Equations. Studies in Nonlinear Sciences, 2, 140-144.

[40] Ma, W.X. (2013) Bilinear Equations, Bell Polynomials and Linear Superposition Principal. Journal of Physics Conference Series, 411, 12021. https://doi.org/10.1088/1742-6596/411/1/012021

[41] Liu, J.G., Du, J.Q., Zeng, Z.F. and Nie, B. (2017) New Three-Wave Solutions for the $(3+1)$-Dimensional Boiti-Leon-Manna-Pempinelli Equation. Nonlinear Dynamics, 88, 655-661. https://doi.org/10.1007/s11071-016-3267-2 